\title{
The expression sequence tag is an effective method for screening DNA segments that predict urinary bladder transitional cell carcinoma prognosis
}

\author{
This article was published in the following Dove Press journal: \\ OncoTargets and Therapy \\ 30 September 2014 \\ Number of times this article has been viewed
}

\author{
Pei-Shan Yang ${ }^{1, *}$ \\ Yu-Chao Hsu',2,* \\ Yu-Hsiang Lin ${ }^{1,2}$ \\ Cheng-Pang Hou ${ }^{1,2}$ \\ Chien-Lun Chen ${ }^{1,2}$ \\ Phei-Lang Chang ${ }^{1-3}$ \\ Horng-Heng Juang ${ }^{3,4}$ \\ Ke-Hung Tsuil ${ }^{1-3}$ \\ 'Department of Urology, Chang \\ Gung Memorial Hospital at \\ Linkou, Taiwan, Republic of China; \\ ${ }^{2}$ School of Medicine, Chang Gung \\ University, Taiwan, Republic of China; \\ ${ }^{3}$ Bioinformation Center, Chang Gung \\ Memorial Hospital, Taiwan, Republic \\ of China; ${ }^{4}$ Department of Anatomy, \\ School of Medicine, Chang Gung \\ University, Kwei-Shan, Tao-Yuan, \\ Taiwan, Republic of China \\ *These authors contributed equally to \\ this work
}

Correspondence: Ke-Hung Tsui

Department of Urology,

Chang Gung Memorial Hospital at Linkou,

Chang Gung University,

5 Fu-Shing Street, Kwei-Shan,

Tao-Yuan 333, Taiwan,

Republic of China

Tel +886 332812002137

Fax +886227358775

Email khtsui@yahoo.com
Purpose: We validated the use of expression sequence tags (ESTs) as an effective method of screening for DNA segments that could predict urothelial cell carcinoma and for identifying ESTs with such predictive value.

Patients and methods: From 2004 to 2009, eleven patients were enrolled in this study: six with high-grade bladder carcinoma and five with low-grade bladder carcinoma. ESTs were used to screen for differential gene expression in a high-grade cell line (MGH-U1) and in a premalignant cell line (MGH-U4). Immunohistochemistry and real-time reverse transcriptionpolymerase chain reaction were used to validate the degree of EST expression and the prognostic value of ESTs.

Results: Apoferritin H subunit (FTH1 protein) exhibited increased expression in high-grade bladder carcinoma compared with that seen in low-grade carcinoma. Immunohistochemistry and real-time reverse transcription-polymerase chain reaction both supported the higher expression of FTH1 in high-grade urothelial carcinoma.

Conclusion: ESTs are useful for detecting the FTH1 protein, which predicts the prognosis of patients with bladder carcinoma.

Keywords: expression sequence tag, transitional cell carcinoma, FTH1

\section{Introduction}

Transitional cell carcinoma (TCC) of the bladder is a very common malignant tumor of the urinary tract. Patients who have TCC may have dramatically different prognoses depending on the characteristics of the tumor. Some TCCs are limited to the mucosal layer, even after several recurrences and surgeries, whereas some TCCs progress quickly to high-grade tumors with metastasis to distant organs. Many methods have been suggested to predict the ability of the tumor to invade into peripheral tissue and metastasize. The major problem with bladder carcinoma is the high recurrence of superficial cancer: more than half of superficial tumors recur within 5 years, and $10 \%-20 \%$ of these tumors progress to invasive disease. ${ }^{1-3}$

Expressed sequence tags (ESTs) are short DNA sequences (200-500 nucleotides) generated by sequencing the $5^{\prime}$ and/or $3^{\prime}$ ends of complementary DNA (cDNA), which are subsequently clustered and counted. Analysis of ESTs is a new method of screening for genes that might be associated with differences in prognoses. ${ }^{4,5}$ In this study, using ESTs, we found that apoferritin H subunit (FTH1) was expressed at higher levels in high-grade TCC (MGH-U1 cells) than in low-grade TCC (MGH-U4 cells). 
Using immunohistochemistry (IHC), we further confirmed the increased expression of FTH1 in high-grade TCC tissue compared with low-grade TCC tissue.

In conclusion, our data demonstrate that evaluation of ESTs was an effective way to screen for gene segments associated with TCC grade and prognosis. Using EST analysis, we found that FTH1 was expressed at higher levels in highgrade TCC than in low-grade TCC; these results were also supported by IHC staining of bladder carcinoma tissue.

\section{Material and methods Patient selection}

From 2004 to 2009, a total of eleven patients were enrolled in this study (Table 1), including six patients with highgrade TCC and five patients with low-grade TCC. The tumor grading was classified according to the World Health Organization/International Society of Urological Pathology Consensus Classification of Urothelial (Transitional Cell) Neoplasms of the Urinary Bladder, published in $1998 .{ }^{6}$ Surgical intervention depended on the characteristics of the patient and aimed at removing the tumor. Six patients underwent transurethral resection of bladder tumor, four patients underwent radical cystectomy, and one patient underwent nephroureterectomy for a renal pelvis tumor. Tissues were harvested from specimens within 30 minutes after surgical removal, placed in 10\% formaldehyde, and frozen in liquid nitrogen for further studies.

\section{Cell lines and cell culture}

MGH-U1 cells were previously established from a bladder tumor $^{3,6}$ and represent high-grade bladder cancer, whereas MGH-U4 cells were previously derived from a man with bladder carcinoma in situ and severe atypia of the bladder, ${ }^{3,6}$ and therefore represent low-grade bladder cancer. EST studies were conducted with both MGH-U1 and MGH-U4 cells. Both cell lines were grown in Roswell Park Memorial Institute (RPMI) 1640 medium supplemented with 10\% fetal bovine serum, $50 \mathrm{U} / \mathrm{mL}$ penicillin, and $50 \mu \mathrm{g} / \mathrm{mL}$ streptomycin in a humidified incubator at $37^{\circ} \mathrm{C}$ with an atmosphere containing $5 \% \mathrm{CO}_{2}$.

\section{$\mathrm{IHC}$}

To detect the expression of FTH1, we used a commercially available anti-FTH1 antibody from Sigma-Aldrich (St. Louis, MO,USA). Paraffin-embedded slides were washed three times with xylene and three times each with 100\%, 95\%, and $70 \%$ ethanol to remove paraffin. Sections were then incubated overnight at $4{ }^{\circ} \mathrm{C}$ with anti-FTH1 antibodies at a 1:100 dilution. The next day, the sections were rinsed and incubated with the appropriate horseradish peroxidaselabeled secondary antibodies (1:1,000 dilution) for 2 hours at room temperature. The slides were then rinsed and prepared for further microscopic study.

\section{EST analysis}

MGH-U4 and MGH-U1 cells were obtained from American Type Culture Collection. MGH-U4 (stage 0, grade atypia, HLA-ABC 3+) and MGH-U1 (stage B, grade 3, HLA-ABC $3+)$ cells are model cell lines for early- and late-stage bladder carcinoma. Total RNA was extracted from harvested cells using a commercial kit (Pharmacia, Sweden), and the residual genomic DNA was hydrolyzed with DNase I. PolyA+ RNA was isolated using the PolyA+ 131 tract mRNA isolation kit (Promega, Madison, WI, USA).

cDNA primed with oligo-dT was synthesized using a ZAP-cDNA synthesis kit and directionally cloned into the EcoRI and XhoI sites of Uni-ZAP XR (Stratagene, CA, USA) vector. Plasmids were excised from the cDNA

Table I Demographic data of TCC patients

\begin{tabular}{|c|c|c|c|c|c|}
\hline Number & Sex & Age, years & Tumor grade & TNM classification & Operative methods \\
\hline THI & M & 48 & High grade & TINOMO & Transurethral resection of bladder tumor \\
\hline $\mathrm{TH} 2$ & M & 81 & High grade & T2N0M0 & Radical cystectomy \\
\hline $\mathrm{TH} 3$ & M & 82 & High grade & T3NOMO & Radical cystectomy \\
\hline TH4 & M & 75 & High grade & TaNIMI & Transurethral resection of bladder tumor \\
\hline TH5 & $\mathrm{F}$ & 83 & High grade & T3NIMI & Transurethral resection of bladder tumor \\
\hline TH6 & M & 69 & High grade & TINOMO & Radical cystectomy \\
\hline TLI & M & 50 & Low grade & TaNOMO & Radical cystectomy \\
\hline TL2 & $\mathrm{F}$ & 78 & Low grade & TaNOMO & Transurethral resection of bladder tumor \\
\hline TL3 & M & 83 & Low grade & TaNOMO & Nephroureterectomy \\
\hline TL4 & M & 70 & Low grade & TaNOMO & Transurethral resection of bladder tumor \\
\hline TL5 & M & 69 & Low grade & TaNOMO & Transurethral resection of bladder tumor \\
\hline
\end{tabular}

Notes: Six patients had high-grade TCC, whereas five patients had low-grade TCC.

Abbreviations: TH, high-grade tumor; TNM, tumor, node, metastasis; TL, low-grade tumor; M, male; F, female; TCC, transitional cell carcinoma. 
libraries using a helper phage and transformed into Escherichia coli DH10B (Invitrogen, Carlsbad, CA, USA), as described by the manufacturer of the kit (Stratagene). Single-pass sequencing of the $5^{\prime}$-end of the cDNA fragment was carried out with $\mathrm{T} 3$ primers on an ABI3730 sequencer (Life Technologies, India). The sequencing trace files obtained were examined by the Phred algorithm for base calling with default parameters. ${ }^{1}$ The quality reads were assembled using the CLC Genomics Suite (CLC Biosciences, Aarhus, Denmark). Annotations were performed via BLAST search to identify similarities between the assembled contigs and sequences deposited in the National Center for Biotechnology Information nonredundant protein database and Swiss-Prot database. ${ }^{7}$ Sequences with an e-value of 10E-10 or less were considered to have significant sequence homology with known genes.

\section{Results EST results}

Approximately 5,000 clones were randomly selected from each library for sequencing. A total of 4,804 and 4,713 ESTs from MGH-U1 and MGH-U4 libraries, respectively, passed the Phred parameters for further analysis. To annotate the ESTs, Basic Local Alignment Search Tool for nucleotides (BLASTn) was used to compare unique ESTs with known genes. Sequences with an E-value of $10 \mathrm{E}-5$ or less were considered to identify known genes or have partial similarity to known genes. Functional domains/motifs and signatures of protein families were identified by comparison with the Interpro and Pfam databases. The specific set of genes unique to early (MGH-U4) and late (MGH-U1) stages was determined by clustering the two data sets together. Among 9,517 unique genes, only 477 genes were expressed in both libraries. Of these genes, $44.86 \%$ were expressed at the same level in both cell lines, and $27.67 \%$ and $27.46 \%$ of the genes were upregulated in MGH-U1 and MGH-U4 cells, respectively.

We used ESTs to evaluate gene expression in MGH-U1 and MGH-U4 cells and found several genes that were strongly expressed in MGH-U1 and MGH-U4 cells (Table 2). The expression density seemed to be correlated with the severity of malignancy.

FTH1 was the first protein identified in this study. FTH1 was expressed in MGH-U1 and MGH-U4 cells, but not in normal tissue. IHC analysis showed that FTH1 expression was different in normal tissues and cancer cells, including MGH-U1 and MGH-U4 cells (Figure 1). In patients with high-grade TCC, FTH1 was expressed at higher levels
Table 2 Genes identified by expression sequence tag

\section{Gene}

Apoferritin $\mathrm{H}$ subunit (FTHI protein)

Transforming growth factor beta-inducible early growth response (zinc finger transcription factor)

Prostate differentiation factor

Plasminogen activator, urokinase preproprotein

Transmembrane 4 superfamily member I (tumor-associated antigen L6; membrane component, surface marker I)

Profilin I; profilin-I (Homo sapiens)

Ribosomal protein SI3; 40S ribosomal protein SI3 (Homo sapiens)

Ribosomal protein LI8a; 60S ribosomal protein LI8a (Homo sapiens)

Hypothetical protein XP_375434

Beta-galactosidase binding lectin precursor; galectin

Notes: The top ten genes were identified by expression sequence tags through comparison of the gene expression frequency between MGH-UI and MGH-U4 cell lines. These ten genes were more frequently found in MGH-UI cells, from a highgrade transitional cell carcinoma cell line, than in MGH-U4 cells, from a low-grade transitional cell carcinoma cell line.

than in patients with low-grade TCC, as demonstrated by densimetric analysis of IHC images (mean measurements, $138 \pm 11$ versus $99 \pm 11$, respectively; $P=0.0346$ ).

FTH1 mRNA expression was detected in both MGH-U1 and MGH-U4 cells. FTH1 expression was higher in MGH-U1 cells than in MGH-U4 cells (mean expression, $35.80 \pm 9.4$ versus $31.14 \pm 6.7$, respectively; $P=0.718$ ), but the difference was not significant.

To evaluate the relationship between FTH1 expression and degree of malignancy, we used real-time reverse transcription-polymerase chain reaction to calculate FTHI mRNA expression in patient tissue samples. FTH1 was expressed at higher levels in patients with high-grade TCC than in patients with low-grade TCC (mean expression, $58.67 \pm 6.125$ versus $24.60 \pm 9.453$, respectively; $P=0.0122$; Figure 2).

Furthermore, several other genes also exhibited differential expression between MGH-U1 and MGH-U4 cells.

\section{Discussion}

Bladder TCC is the sixth most prevalent malignancy in men worldwide. From 1985 to 2005, the number of bladder cancers diagnosed in the United States increased by more than 50\%, and from 1975 to 1996 , the 5-year survival rate for those diagnosed with bladder cancer increased from $75 \%$ to $81 \%$. $^{8}$

Most pT1 tumors of the bladder carcinoma are low- or high-grade papillary TCCs, whereas the more deeply invasive pT2-pT4 TCCs are nonpapillary and high-grade. Several studies have shown that pT1 tumors have a less favorable prognosis than pTa neoplasms. However, pTa 


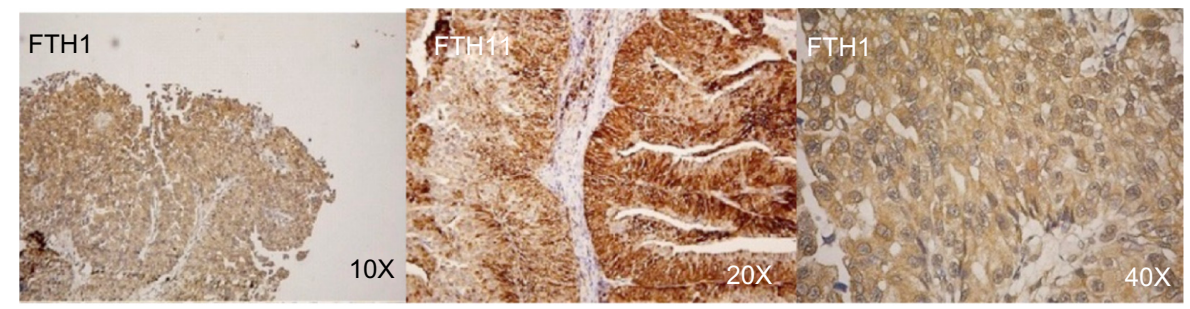

Densimetry in IHC (FTH1)

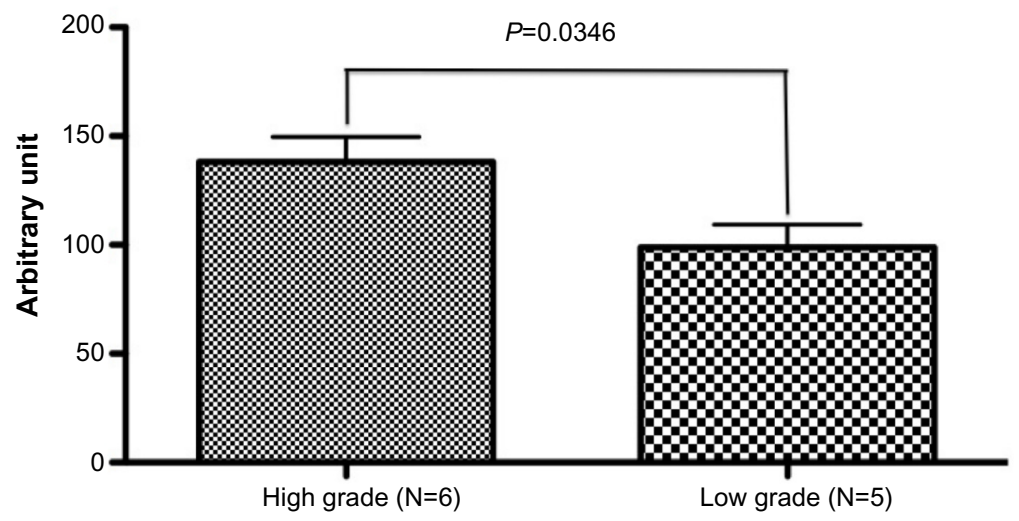

Figure I Urothelial specimens from TCC patients were all positive for FTHI by IHC.

Note: FTHI was expressed at higher levels in patients with high-grade TCC than in patients with low-grade TCC $(P=0.0346)$.

Abbreviations: FTHI, apoferritin $\mathrm{H}$ subunit; IHC, immunohistochemistry; TCC, transitional cell carcinoma.

A
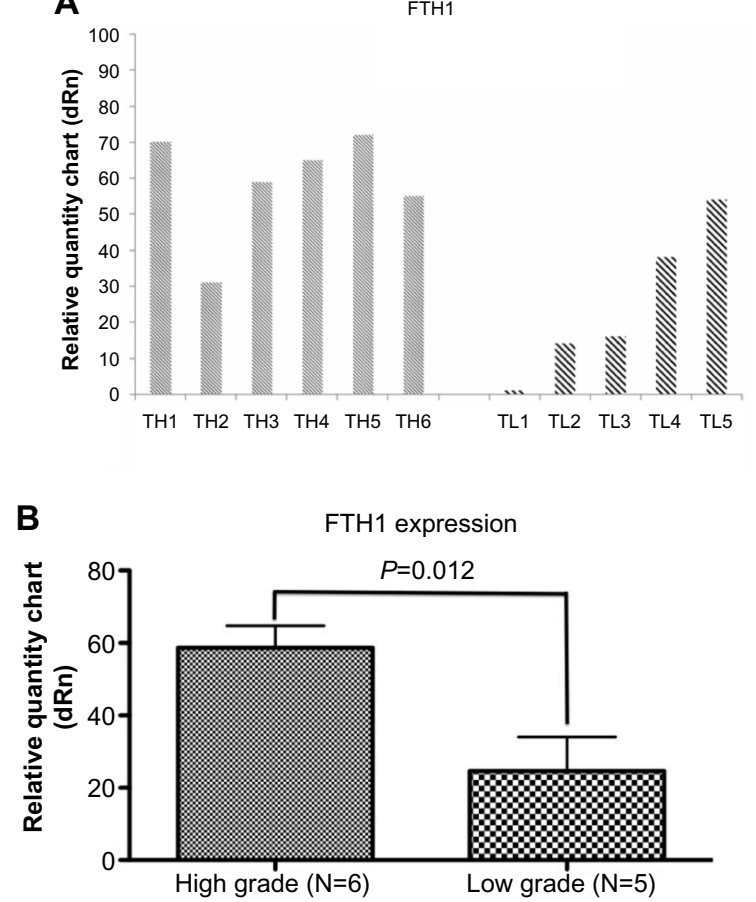

Figure 2 Reverse transcriptase-polymerase chain reaction analysis of tissue samples from six patients with high-grade TCC and five patients with low-grade TCC showed that FTHI was expressed at higher levels in patients with high-grade TCC than in patients with low-grade TCC $(P=0.0122)$

Notes: (A) Reverse transcriptase-polymerase chain reaction analysis of tissue samples from six patients with high-grade TCC and five patients with low-grade TCC. (B) The RT-PCR showed that FTHI was expressed at higher levels in patients with high-grade TCC than in patients with low-grade TCC $(P=0.0122)$.

Abbreviations: $\mathrm{FTHI}$, apoferritin $\mathrm{H}$ subunit; $\mathrm{TH}$, high-grade tumor; $\mathrm{TL}$, low-grade tumor; dRn, delta ribonucleotide; TCC, transitional cell carcinoma. and $\mathrm{pT} 1$ neoplasms are grouped together because both $\mathrm{pTa}$ and $\mathrm{pT} 1$ lesions are managed conservatively.

The cause of death in bladder cancer patients is usually not the malignancy itself, but the consequences of tumor recurrence.

Non-muscle-invasive bladder cancer can be managed by transurethral resection, followed by regular cystoscopies. However, if the bladder cancer is found to invade the muscle, then radical cystectomy with urinary conduits is the standard treatment. ${ }^{9}$ Changes in health-related quality of life in patients treated with radical cystectomy and urinary diversion, with regard to urinary, bowel, and sexual functions, as well as body image perception, can be prolonged and dramatic. ${ }^{10}$ Therefore, the identification of biomarkers that predict the prognosis of TCC is crucial.

In this study, we used ESTs to identify the cluster presentation of genes from MGH-U1 and MGH-U4 cells. MGH-U1 and MGH-U4 cells were derived from tumors exhibiting different degrees of malignancy and may represent cells at different stages of tumor progression, with MGH-U1 cells representing highly malignant cells and MGH-U4 cells representing mildly malignant, possibly premalignant, cells. ${ }^{11-13}$ We have found that FTH1 expression was highest in MGH-U1 cells. To demonstrate the relationship between FTH1 protein expression and the degree of malignancy, we conducted a study to quantify mRNA expression via real-time reverse transcription-polymerase 
chain reaction and then correlated the expression data with recurrence and survival in patients with bladder carcinoma. We found that FTH1 was expressed at higher levels in patients with high-grade bladder carcinoma than in patients with low-grade bladder carcinoma. Therefore, FTH1 may be a biomarker for predicting prognosis in bladder carcinoma.

\section{Conclusion}

In this study, we were able to use ESTs to identify differentially expressed genes, such as FTH1, in low- and high-grade urothelial carcinoma. IHC was used to confirm FTH1 expression, whereas real-time reverse transcription-polymerase chain reaction was used to quantify FTH1 expression. Thus, FTH1 was a possible predictive marker for bladder carcinoma prognosis.

\section{Acknowledgments}

This research was supported by research grants from the Chang Gung Memorial Hospital (CMRPG381681, CMRPG392142, CMRPG3B1892) and the National Science Council, Taiwan, Republic of China (101-2314-B-182A-099-MY3).

\section{Disclosure}

The authors report no conflicts of interest in this work.

\section{References}

1. Ewing B, Green P. Base-calling of automated sequencer traces using phred. II. Error probabilities. Genome Res. 1998;8(3):186-194.
2. Ewing B, Hillier L, Wendl MC, Green P. Base-calling of automated sequencer traces using phred. I. Accuracy assessment. Genome Res. 1998;8(3):175-185.

3. Lin CY, Tsui KH, Yu CC, Yeh CW, Chang PL, Yung BY. Searching cell-secreted proteomes for potential urinary bladder tumor markers. Proteomics. 2006;6(15):4381-4389.

4. Badawi AF. Molecular and genetic events in schistosomiasisassociated human bladder cancer: role of oncogenes and tumor suppressor genes. Cancer Lett. 1996;105(2):123-138.

5. Feichtinger J, McFarlane RJ, Larcombe LD. CancerEST: a web-based tool for automatic meta-analysis of public EST data. Database (Oxford). 2014;2014(0):bau024.

6. Kegye A, Naszály A. Radiotherapy for lung metastases in a patient with ewing sarcoma. Sarcoma. 1998;2(3-4):209-213.

7. Altschul SF, Gish W, Miller W, Myers EW, Lipman DJ. Basic local alignment search tool. J Mol Biol. 1990;215(3):403-410.

8. Jemal A, Siegel R, Xu J, Ward E. Cancer statistics, 2010. CA Cancer J Clin. 2010;60(5):277-300.

9. Herr HW. Transurethral resection of muscle-invasive bladder cancer: 10-year outcome. J Clin Oncol. 2001;19(1):89-93.

10. Aboumohamed AA, Raza SJ, Al-Daghmin A, et al. Health-related quality of life outcomes after robot-assisted and open radical cystectomy using a validated bladder-specific instrument: a multi-institutional study. Urology. 2014;83(6):1300-1308.

11. Lin CW, Lin JC, Prout GR Jr. Establishment and characterization of four human bladder tumor cell lines and sublines with different degrees of malignancy. Cancer Res. 1985;45(10):5070-5079.

12. Tsui KH, Tang P, Lin CY, Chang PL, Chang CH, Yung BY. Bikunin loss in urine as useful marker for bladder carcinoma. J Urol. 2010;183(1): 339-344.

13. Tsui KH, Juang HH, Lee TH, Chang PL, Chen CL, Yung BY. Association of nucleophosmin/B23 with bladder cancer recurrence based on immunohistochemical assessment in clinical samples. Acta Pharmacol Sin. 2008;29(3):364-370.
OncoTargets and Therapy

\section{Publish your work in this journal}

OncoTargets and Therapy is an international, peer-reviewed, open access journal focusing on the pathological basis of all cancers, potential targets for therapy and treatment protocols employed to improve the management of cancer patients. The journal also focuses on the impact of management programs and new therapeutic agents and protocols on

\section{Dovepress}

patient perspectives such as quality of life, adherence and satisfaction. The manuscript management system is completely online and includes a very quick and fair peer-review system, which is all easy to use. Visit http://www.dovepress.com/testimonials.php to read real quotes from published authors. 